\title{
Adaptation to tRNA acceptor stem structure by flexible adjustment in the catalytic domain of class I tRNA synthetases
}

\author{
CUIPING LIU, JEFFREY M. SANDERS, JOHN M. PASCAL, and YA-MING HOU ${ }^{1}$ \\ Thomas Jefferson University Department of Biochemistry and Molecular Biology, Philadelphia, Pennsylvania 19107, USA
}

\begin{abstract}
Class I aminoacyl-tRNA synthetases (aaRSs) use a Rossmann-fold domain to catalyze the synthesis of aminoacyl-tRNAs required for decoding genetic information. While the Rossmann-fold domain is conserved in evolution, the acceptor stem near the aminoacylation site varies among tRNA substrates, raising the question of how the conserved protein fold adapts to RNA sequence variations. Of interest is the existence of an unpaired C-A mismatch at the 1-72 position unique to bacterial initiator tRNA $^{\text {fMet }}$ and absent from elongator tRNAs. Here we show that the class I methionyl-tRNA synthetase (MetRS) of Escherichia coli and its close structural homolog cysteinyl-tRNA synthetase (CysRS) display distinct patterns of recognition of the 1-72 base pair. While the structural homology of the two enzymes in the Rossmann-fold domain is manifested in a common burst feature of aminoacylation kinetics, CysRS discriminates against unpaired 1-72, whereas MetRS lacks such discrimination. A structurebased alignment of the Rossmann fold identifies the insertion of an $\alpha$-helical motif, specific to CysRS but absent from MetRS, which docks on 1-72 and may discriminate against mismatches. Indeed, substitutions of the CysRS helical motif abolish the discrimination against unpaired 1-72. Additional structural alignments reveal that with the exception of MetRS, class I tRNA synthetases contain a structural motif that docks on 1-72. This work demonstrates that by flexible insertion of a structural motif to dock on 1-72, the catalytic domain of class I tRNA synthetases can acquire structural plasticity to adapt to changes at the end of the tRNA acceptor stem.
\end{abstract}

Keywords: Rossmann-fold domain; aminoacylation; burst kinetics; docking on tRNA

\section{INTRODUCTION}

Aminoacyl-tRNA (aa-tRNA) synthetases (aaRSs) are essential enzymes that catalyze amino acid attachment to the $3^{\prime}$ end of cognate tRNAs to provide a physical link to relate the amino acid with the tRNA anticodon sequences. These aaRSs are divided into two structurally distinct classes based on the specific features of the catalytic domain (Ibba and Soll 2000). The class I catalytic domain uses the wellcharacterized Rossmann dinucleotide-binding fold to catalyze the ATP-dependent activation of amino acids and the transfer of the activated aminoacyl-adenylates to the CCA sequence at the tRNA $3^{\prime}$ end. Enzymes with the class I catalytic domain include those that specify Met, Cys, Arg, Ile, Leu, Val, Glu, Gln, Tyr, and Trp. The remaining en-

\footnotetext{
${ }^{1}$ Corresponding author.

E-mail ya-ming.hou@jefferson.edu.

Article published online ahead of print. Article and publication date are at http://www.rnajournal.org/cgi/doi/10.1261/rna.029983.111.
}

zymes belong to class II, which instead employ three degenerate motifs to coordinate ATP binding in an anti-parallel core of $\beta$ strands. With few exceptions (Ibba et al. 1997), the classification of tRNA synthetases is conserved in evolution. In both classes, the catalytic domain is fused to tRNAdocking domains, which select tRNA for aminoacylation by discrimination among specific nucleotide determinants.

A major tRNA-docking domain, responsible for recognition of the anticodon, is localized at the opposite end from the active site. Upon anticodon binding, this domain conducts long-range communication with the active-site domain to modulate the specificity of aminoacylation (Uter and Perona 2004). The anticodon-binding domain is not well conserved, even among tRNA synthetases of the same class, which permits the development of specific domain structures tailored to the variable motifs that contact the anticodon sequences of cognate tRNAs. In contrast, the other major tRNA-docking domain, responsible for recognition of the acceptor end, is proximal to the active site and most often exists as an insertion into the conserved 
catalytic domain. However, the fundamental challenge to understand acceptor stem recognition is how the conserved catalytic domain accommodates the insertion in ways that allow specific recognition of acceptor stem sequences, which vary not only in nucleotide sequences but also in the sequence-dependent structural conformation of the 3' end (Lee et al. 1993; Puglisi et al. 1994; Hou et al. 1998).

An example that illustrates the challenge to understand acceptor stem recognition by aaRSs is found in class I: While Escherichia coli $(E c)$ methionyl-tRNA synthetase (MetRS) and $E$. coli $(E c)$ cysteinyl-tRNA synthetase (CysRS) are highly homologous throughout the entire class I structure (Hou et al. 1991; Hauenstein et al. 2004; Nakanishi et al. 2005), these two enzymes recognize tRNA substrates with distinct acceptor-end nucleotides and structures (Fig. 1). EcMetRS must accommodate both the initiator tRNA $^{\text {fMet }}$ and the elongator tRNA ${ }^{\text {Met }}$ for aminoacylation, which possess, respectively, a C-A and G-C base pair at the 1-72 position adjacent to the $3^{\prime}$ end, while sharing A73 in common as an important discriminator base for aminoacylation (Martinis and Schimmel 1992). The unpaired C-A at 1-72 is unusual and is unique to the initiator tRNA ${ }^{\mathrm{fMet}}$ in bacterial species. Mutational analysis has suggested that the combination of C-A at 1-72 and A73 in the initiator tRNA is responsible for the unusual bent conformation of the $3^{\prime}$ end, where the terminal A76 folds back toward the $5^{\prime}$ end, whereas the combination of G-C at 1-72 and A73 in the elongator tRNA ${ }^{\mathrm{Met}}$ confers a straight conformation of the $3^{\prime}$ end (Puglisi et al. 1994). In contrast, CysRS recognizes only one isoacceptor, which possesses a G-C base pair at 172 , followed by a conserved but unusual U73 that plays a key role in aminoacylation (Liu et al. 2007, 2011). Mutational and structural analysis has also implicated U73 in modulating the structure of the $3^{\prime}$ end (Hou et al. 1998, 2001). The wide disparity in nucleotides and structures at the acceptor end among the three tRNAs, EctRNA ${ }^{\mathrm{fMet}}, E_{\mathrm{ctRNA}}{ }^{\mathrm{Met}}$, and $E c t R N A{ }^{\text {Cys }}$, highlights the challenge of recognition by a conserved catalytic domain.

Here we address this challenge by using single turnover assays to monitor acceptor stem recognition with the focus on the enzymatic steps that involve the chemistry of aminoacylation. Work in our laboratory and others has demonstrated that for several class I tRNA synthetases (such as CysRS, ValRS, IleRS, and GlnRS), the enzymatic steps beginning with substrate binding up to and including the chemistry of aminoacylation are fast relative to the steps involved in the release of aa-tRNA (Eldred and Schimmel 1972; Uter and Perona 2004; Zhang et al. 2006). This observation suggests that steady-state analysis of aminoacylation in multiple turnovers would be dominated by the slow release after aminoacylation and would be unable to capture the essence that occurs during aminoacylation. In contrast, single turnover assays based on chemical-quench methodologies are capable of isolating the equilibrium and rate constants that occur during the chemistry of aminoacylation. The application of single turnover assays to monitor aminoacylation by both EcMetRS and EcCysRS has allowed us to compare the two enzymes in the recognition of tRNA acceptor end. This comparison, together with structural alignment and modeling of the Rossmann fold, has identified the insertion of an $\alpha$-helical motif to the Rossmann fold of EcCysRS that is important for the stringent discrimination against 1-72 mismatches. This insertion is absent from the Rossmann fold of EcMetRS, providing a basis for the relaxed recognition of 1-72. Additional structural alignments confirmed that with the exception of MetRS, many class I tRNA synthetases contain an insertion of a specific structural motif that docks on 1-72. Thus, the flexible adjustment of an insertion motif in the conserved Rossmann fold of class I enzymes can provide a mechanism to accommodate distinct features in the tRNA acceptor end.

\section{RESULTS}

\section{Kinetic analysis of EcMetRS}

Although EcMetRS shares high homology with EcCysRS in both sequence and high-resolution X-ray structure (Hou et al. 1991; Hauenstein et al. 2004; Nakanishi et al. 2005), we tested whether this high homology was manifested in similar kinetics, which would serve as an independent probe for the correlation between structure and activity. An important feature of aminoacylation by EcCysRS was the appearance of burst kinetics in pre-steady-state conditions, where the rate of aminoacylation in the first turnover was fast $\left(14 \pm 1 \mathrm{sec}^{-1}\right)$, followed by steady-state accumulation of the product aa-tRNA at a much slower rate $\left(2.5 \pm 0.06 \mathrm{sec}^{-1}\right)$ (Zhang et al. 2006). The appearance of burst kinetics indicated that the chemistry steps required for aminoacylation were fast relative to the release of aa-tRNA. In contrast, aminoacylation by several class II aaRSs was linear with time, exhibiting no burst kinetics (Guth et al. 2005; Zhang et al. 2006). This suggests that the distinct kinetic features

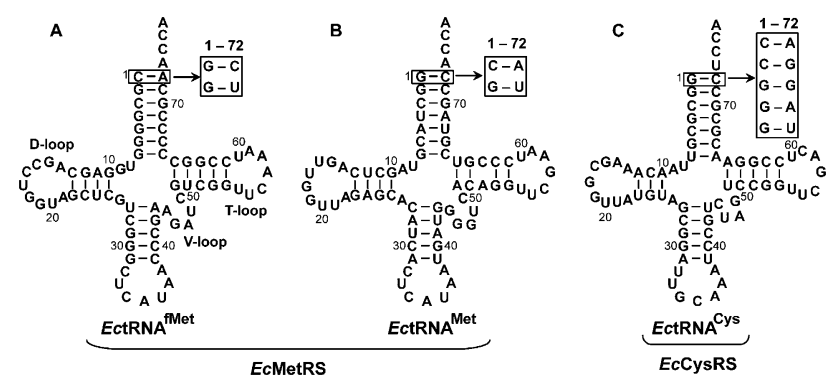

FIGURE 1. Sequences and cloverleaf structures of tRNA. (A) $E c t R N A^{\mathrm{fMet}}$, (B) EctRNA ${ }^{\mathrm{Met}}$, and $(C) E c t$ RNA ${ }^{\text {Cys }}$. The 1-72 base pair of the tRNA acceptor stem is boxed, and substitutions of the base pair are indicated by arrows. 
of the two classes are manifests of their distinct catalytic structures. To test this prediction further, we determined if aminoacylation of EcMetRS under pre-steady-state conditions would exhibit burst kinetics. The enzyme was purified as a His-tag fusion from an overexpression strain of E. coli. The substrate initiator $E c t R N A^{\mathrm{fMet}}$ and elongator $E c t R N A^{\text {Met }}$ were both synthesized by in vitro transcription based on template sequences reconstituted from overlapping oligonucleotides. Transcription of EctRNA ${ }^{\mathrm{fMet}}$ with the unfavorable initiation $\mathrm{C} 1$ nucleotide was achieved by the synthesis of a longer precursor transcript with a hammerhead ribozyme fused to the $5^{\prime}$ end of the tRNA sequence, allowing the ribozyme to self-cleave after transcription to release the mature tRNA transcript (Fechter et al. 1998). Both the initiator and elongator transcripts were refolded by annealing and were verified as substrates for EcMetRS by demonstrating a capacity for aminoacylation to $\sim 50 \%$ in extended time courses.

Concentrations of EcMetRS ( $1 \mu \mathrm{M}$ final) and each tRNA substrate $(10 \mu \mathrm{M}$ final) were adjusted to allow monitoring of a single turnover on the enzyme, followed by steady-state synthesis of aa-tRNA. Aminoacylation was performed on the rapid chemical quench RQF3, in which EcMetRS in the reaction buffer was incubated in one syringe and was rapidly mixed with tRNA, radiolabeled-Met, and ATP in the second syringe. Time-dependent sampling of aminoacylation was quenched by acid precipitation (with trichloroacetic acid [TCA]) on filter pads, which were analyzed by scintillation counting for the attachment of labeled Met to tRNA. A set of mixing controls established that the observed reaction rates were independent of the premixing of the reactants. Additionally, because EcMetRS has an ability to self-methionylate to lysine residues near the active site (Gillet et al. 1997), as evidenced in the self-labeling by covalent incorporation of ${ }^{35} \mathrm{~S}$-Met to the enzyme in both the presence and absence of tRNA ${ }^{\text {Met }}$ (Supplemental Fig. S1A,B), aminoacylation was also monitored by two different methods. In one, aminoacylation with ${ }^{35} \mathrm{~S}$-Met was quenched with $5 \mathrm{mM}$ cold Met, followed by acid precipitation on filter pads, and in the other, aminoacylation was performed on ${ }^{32} \mathrm{P}$-labeled A76 of tRNA $^{\text {Met }}$, followed by S1 digestion to single nucleotides and by analysis of methionyl-A76 versus A76 on a TLC plate (Wolfson et al. 1998). Single turnover analysis showed that the kinetics of aminoacylation catalyzed by $2 \mu \mathrm{M}$ $E c$ MetRS were virtually identical among the three methods (TLC, TCA washes without Met, and TCA washes with Met), showing a rate constant of 11.7, 12.4 , and $11.7 \mathrm{sec}^{-1}$, respectively (Supplemental Fig. S1C,D). The consistency among the different assays suggests that despite the selfmethionylation by the enzyme, the kinetics of aminoacylation in the presence of tRNA is unaffected. This is consistent with the notion that the self-methionylation reaction occurs at a much slower rate relative to aminoacylation (Gillet et al. 1997), so that the self-methionylation was not detected in the time scale of one turnover of aminoacylation.

We focused on the assay of acid precipitation in the absence of Met. Analysis of the time course of aminoacylation indeed revealed that for both tRNA substrates, a rapid burst of aminoacylation appeared in the first enzyme turnover, followed by a slower and linear steadystate conversion (Fig. 2). For the initiator $E_{c t R N A}{ }^{\mathrm{fMet}}$, fitting the data to a burst equation yielded the rate constant in the first turnover $k_{\mathrm{chem}}\left(30 \pm 7 \mathrm{sec}^{-1}\right)$ and the subsequent steady-state rate constant $k_{\text {cat }}\left(3.7 \pm 0.3 \mathrm{sec}^{-1}\right)$, confirming that the chemistry is fast relative to product release by about eightfold (Fig. 2A). The $k_{\text {chem }}$ was a composite term that represented the chemistry in the slower of the two-step aminoacylation reaction, which is most likely the aminoacyl-transfer step (Zhang et al. 2006). Similarly, fitting the data of the elongator EctRNA ${ }^{\text {Met }}$ to a burst equation yielded $k_{\mathrm{chem}}\left(41 \pm 8 \mathrm{sec}^{-1}\right)$ and $k_{\text {cat }}$ $\left(8.8 \pm 1 \mathrm{sec}^{-1}\right)$, showing a faster rate constant in the first turnover than the subsequent steady-state constant by about fivefold (Fig. 2B). While the two sets of data differed, likely reflecting differences in the acceptor end of the two tRNAs, both supported the notion that the synthesis of aa-tRNA $\left(k_{\text {chem }}\right)$ occurs fast and that the rate-limiting step $\left(k_{\text {cat }}\right)$ takes place after aminoacylation rather than during the chemistry steps in the active site. Although a previous analysis of EcMetRS failed to show burst kinetics (Mulvey and Fersht 1978), it was likely attributed to the delayed sampling (beginning at $0.1 \mathrm{sec}^{-1}$ ) rather than the sampling performed here (beginning at $0.003 \mathrm{sec}^{-1}$ ). The identifica-
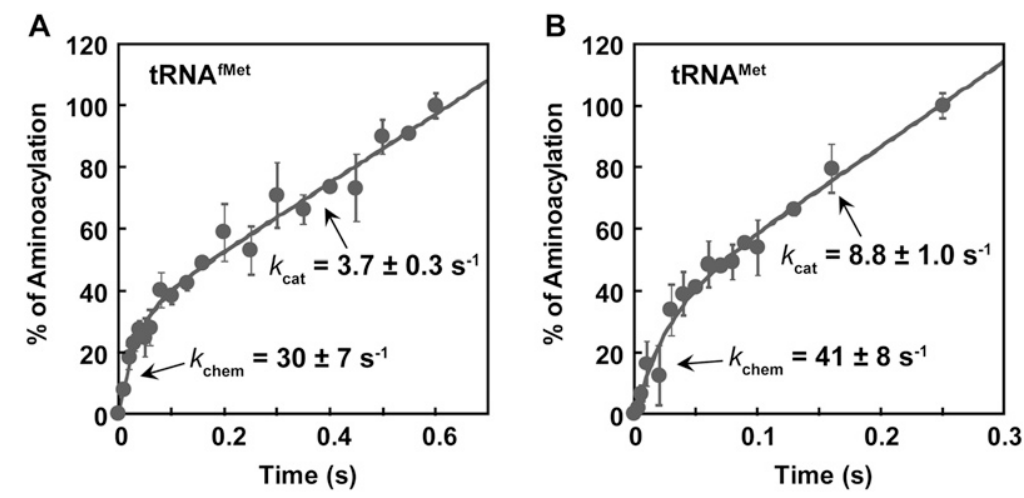

FIGURE 2. Pre-steady-state aminoacylation of $E c$ MetRS. (A) A representative set of data from the average of two experiments of aminoacylation over time with $1 \mu \mathrm{M} E c \mathrm{MetRS}$ and $10 \mu \mathrm{M}$ $E_{c t R N A}{ }^{\text {fMet }}$. (B) A representative set of data from the average of two experiments of aminoacylation over time with $1 \mu \mathrm{M} E c$ MetRS and $10 \mu \mathrm{M} E_{c t R N A}{ }^{\mathrm{Met}}$. Rate constants $k_{\text {chem }}$ and $k_{\text {cat }}$ are shown in each time course. Errors: SDs. 
tion of burst kinetics recapitulate the feature of EcCysRS, demonstrating that the structural homology in the active site between $E c$ MetRS and EcCysRS is functionally significant and is manifested in the kinetics of aminoacylation.

\section{Mutational analysis of acceptor end}

$E_{c t R N A}{ }^{\mathrm{fMet}}$ and EctRNA ${ }^{\mathrm{Met}}$ contain C-A and G-C at 1-72, respectively, whereas EctRNA ${ }^{\text {Cys }}$ contains G-C (Fig. 1). To determine if the 1-72 base pair is a determinant for aminoacylation by EcMetRS and EcCysRS, we created nucleotide substitutions at 1-72 in the three tRNAs. The C-A in tRNA $^{\mathrm{fMet}}$ was replaced with the stronger base pairs G-C and $\mathrm{G}-\mathrm{U}$ based on $\mathrm{H}$-bonding potential; the $\mathrm{G}-\mathrm{C}$ in tRNA $^{\text {Met }}$ was replaced with the weaker base pairs G-U and $\mathrm{C}-\mathrm{A}$; and the G-C in tRNA ${ }^{\mathrm{Cys}}$ was replaced with both matched (C-G, G-U) and mismatched (C-A, G-G, and G-A) base pairs. Aminoacylation of wild-type (WT) and mutant tRNA transcripts was assayed in single turnover conditions, where the enzyme was in molar excess of the tRNA substrate while ATP and amino acid were saturating relative to the respective $K_{\mathrm{m}}$ values. Time courses of aminoacylation revealed single exponential kinetics at all concentrations of the enzyme (Supplemental Fig. S2), indicating rapid equilibrium binding of enzyme-tRNA-ATP-amino acid complexes. Analysis of $k_{\text {app }}$ as a function of enzyme concentration revealed that all the data were well fit to a hyperbola, providing additional evidence for the rapid equilibrium binding conditions under which the kinetics were measured. This analysis yielded the maximum $k_{\mathrm{obs}}$ of the aminoacylation reaction and $K_{\mathrm{d}}$ of each enzyme for the tRNA substrate. The maximum $k_{\text {obs }}$ was previously interpreted as the chemical rate $\left(k_{\text {chem }}\right)$ of aminoacyl transfer to tRNA by EcCysRS (Zhang et al. 2006), rather than a molecular isomerization step. This was confirmed for EcCysRS by showing that, with WT-tRNA ${ }^{\text {Cys }}$, the $k_{\text {obs }}$ of the two-step aminoacylation reaction and the $k_{\text {obs }}$ of the aminoacyl-trans- fer reaction were virtually identical (13.4 and $12.7 \mathrm{sec}^{-1}$ ), and the same was confirmed with the C1-A72 mutant of tRNA ${ }^{\text {Cys }}$ $\left(1.0\right.$ and $0.7 \mathrm{sec}^{-1}$ ) (Supplemental Fig. S3A,B). Thus, even with mutations in the acceptor end, the chemical transformation rate of aminoacyl transfer was still the limiting factor of the overall rate of aminoacylation. This provided the basis for defining the maximum $k_{\mathrm{obs}}$ as $k_{\mathrm{chem}}$ and for determining the catalytic efficiency $k_{\text {chem }} / K_{\mathrm{d}}$ of each tRNA substrate (Table 1).

Aminoacylation of the initiator $E c t R N A^{\text {fMet }}$ by $E c$ MetRS exhibited $k_{\text {chem }}\left(28 \pm 3 \mathrm{sec}^{-1}\right)$ and $K_{\mathrm{d}}$ (tRNA, $3.6 \pm 0.9$ $\mu \mathrm{M})$, closely similar to $k_{\text {chem }}\left(14 \pm 1 \mathrm{sec}^{-1}\right)$ and $K_{\mathrm{d}}$ (tRNA, $3.2 \pm 0.3 \mu \mathrm{M}$ ) of aminoacylation by EcCysRS (Zhang et al. 2006). The similarity was preserved in the $k_{\text {chem }}(45 \pm 5$ $\mathrm{sec}^{-1}$ ) and $K_{\mathrm{d}}$ (tRNA, $\left.2.4 \pm 0.8 \mu \mathrm{M}\right)$ of the elongator $E_{c t R N A}{ }^{\text {Met }}$ (Table 1). All three reactions displayed $k_{\text {chem }} / K_{\mathrm{d}}$ values in the range of $10^{6}-10^{7} \mathrm{M}^{-1} \mathrm{sec}^{-1}$, indicating high efficiencies for macromolecular interaction. In addition, the $k_{\text {chem }}$ values of $E c t R N A^{\text {fMet }}$ and $E c t R N A^{\text {Met }}$ as determined from the titration experiments were closely similar to the values obtained from the burst kinetics in the pre-steady-state analysis (Fig. 2), indicating the validity of both assays and their complementary support of each other.

The substitution at 1-72 in EctRNA ${ }^{\mathrm{fMet}}$ with G-C had a small effect on $K_{\mathrm{d}}$ (tRNA), while the substitution with G-U had a minor effect on $k_{\text {chem }}$; both substitutions maintained the value of the other parameter, resulting in the loss of $k_{\text {chem }} / K_{\mathrm{d}}$ by only 4.4- and 8.9-fold, respectively. Similarly, substitutions at 1-72 in EctRNA ${ }^{\text {Met }}$ with C-A and with G-U had a small effect on $k_{\text {chem }}$, with little effect on $K_{\mathrm{d}}$, resulting in the loss of $k_{\mathrm{chem}} / K_{\mathrm{d}}$ by 2.7 - and 3.8 -fold, respectively. The magnitudes of these effects are small compared to substitutions at 1-72 in EctRNA ${ }^{\mathrm{Cys}}$ for aminoacylation by EcCysRS. Specifically, EcCysRS was highly sensitive to substitutions of 1-72; both the unpaired C-A and the paired C-G had a major effect on $k_{\text {chem }}$, as well as on $K_{\mathrm{d}}$

TABLE 1. Kinetics of single turnover aminoacylation

\begin{tabular}{|c|c|c|c|c|c|c|}
\hline tRNA & $\begin{array}{l}\text { Nucleotides } \\
\text { at } 1-72\end{array}$ & Enzyme & $k_{\text {chem }}\left(\sec ^{-1}\right)$ & $K_{\mathrm{d}}(\mu \mathrm{M})$ & $k_{\text {chem }} / K_{\mathrm{d}}\left(\mathrm{sec}^{-1} \mathrm{M}^{-1}\right)$ & $\begin{array}{l}\text { Relative activity } \\
\text { to cognate pair }\end{array}$ \\
\hline \multirow[t]{3}{*}{$E C$ tRNA ${ }^{\text {fMet }}$} & C-A (WT) & Ec MetRS & $28 \pm 3$ & $3.6 \pm 0.9$ & $(8 \pm 2) \times 10^{6}$ & 1 \\
\hline & $\mathrm{G}-\mathrm{C}$ & & $22.6 \pm 6$ & $12.6 \pm 3$ & $(1.8 \pm 0.6) \times 10^{6}$ & $1 / 4.4$ \\
\hline & $\mathrm{G}-\mathrm{U}$ & & $3.2 \pm 0.2$ & $3.5 \pm 0.7$ & $(0.9 \pm 0.2) \times 10^{6}$ & $1 / 8.9$ \\
\hline \multirow[t]{3}{*}{ Ec tRNA ${ }^{\mathrm{Met}}$} & G-C (WT) & & $45 \pm 5$ & $2.4 \pm 0.8$ & $(19 \pm 7) \times 10^{6}$ & 1 \\
\hline & C-A & & $24 \pm 2$ & $3.3 \pm 0.9$ & $(7 \pm 2) \times 10^{6}$ & $1 / 2.7$ \\
\hline & $\mathrm{G}-\mathrm{U}$ & & $9.2 \pm 0.8$ & $2.0 \pm 0.6$ & $(5 \pm 1) \times 10^{6}$ & $1 / 3.8$ \\
\hline \multirow[t]{6}{*}{$E_{C}$ tRNA ${ }^{\text {Cys }}$} & $\mathrm{G}-\mathrm{C}(\mathrm{WT})$ & Ec CysRS & $14 \pm 1$ & $3.2 \pm 0.3$ & $(4.4 \pm 0.5) \times 10^{6}$ & 1 \\
\hline & C-A & & $0.5 \pm 0.1$ & $14 \pm 4$ & $(4 \pm 1) \times 10^{4}$ & $1 / 110$ \\
\hline & C-G & & $2.4 \pm 0.6$ & $46 \pm 5$ & $(5 \pm 1) \times 10^{4}$ & $1 / 88$ \\
\hline & $\mathrm{G}-\mathrm{G}$ & & $1.8 \pm 0.3$ & $8.7 \pm 2.5$ & $(2.1 \pm 0.7) \times 10^{5}$ & $1 / 21$ \\
\hline & G-A & & $2.7 \pm 0.1$ & $6 \pm 0.6$ & $(4.5 \pm 0.5) \times 10^{5}$ & $1 / 9.8$ \\
\hline & $\mathrm{G}-\mathrm{U}$ & & $134 \pm 30$ & $25 \pm 3$ & $(5 \pm 1) \times 10^{6}$ & $1 / 0.9$ \\
\hline
\end{tabular}

The error values represent standard error values. 
(tRNA), leading to the loss of $k_{\text {chem }} / K_{\mathrm{d}}$ by 110 - and 88 -fold, respectively. The severe defects, regardless of the nature of pairing, suggest that $\mathrm{C} 1$ is not well tolerated by CysRS. Thus, CysRS has two requirements: G1 and a G1-mediated base pairing interaction at 1-72. In contrast, substitutions of the 72 nucleotide showed variable effects, with the unpaired G-G and G-A showing moderate effects (a loss of $k_{\text {chem }} / K_{\mathrm{d}}$ by 21 - and 9.8 -fold, respectively), while the paired G-U showing a minor effect (0.9-fold).

A graphic analysis of the mutational effects showed that $E c$ MetRS is relatively relaxed with respect to recognition of 1-72 compared with EcCysRS (Fig. 3). The accommodation of both paired and unpaired nucleotides at 1-72 by $E c$ MetRS is consistent with the necessity of this enzyme to recognize both $E c t R N A^{\mathrm{fMet}}$ and EctRNA ${ }^{\text {Met }}$. In contrast, the inability to accommodate variations at 1-72 by EcCysRS indicates a more stringent structural requirement.

\section{An $\alpha$-helical motif in the Rossmann-fold domain}

To elucidate the structural determinant that enables $E c C y s R S$ to discriminate among the base pairing structures of 1-72, we focused on the Rossmann-fold domain of the enzyme in complex with the acceptor stem of the cognate tRNA and performed a structural comparison with the corresponding structure in MetRS (Fig. 4). Of interest was the $\mathrm{CP} 1$ polypeptide, which is typically inserted between the two halves of the Rossmann fold. In the structure of Aquifex aeolicus MetRS (AaMetRS) (Fig. 4A; Nakanishi et al. 2005), which has $>50 \%$ sequence similarity to its $E$. coli counterpart, the CP1 docked on the tRNA acceptor end from the minor groove side, a feature shared in common with other class I aaRSs. In the structure of EcCysRS (Hauenstein et al. 2004), while the CP1 was similarly placed near the minor groove of the tRNA acceptor end, this peptide contained a notable $\alpha$-helical motif that directly docked on the 1-72 base pair (Fig. 4B). Structural alignment showed that while $\mathrm{C} \alpha$ atoms of the two structures in the Rossmann-fold alone (without tRNA) are well aligned (root-mean-square deviation $=2.4 \AA$ based on 593 atoms in the Rossmann fold), the $\alpha$-helical motif in the EcCysRS-tRNA complex occupied a spatial position that

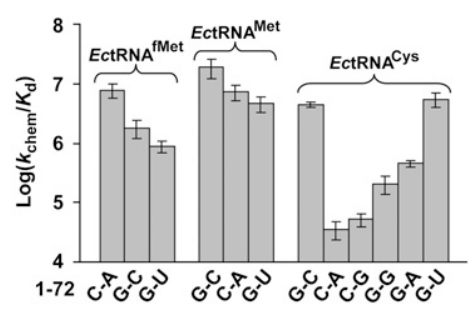

FIGURE 3. Effect of 1-72 base pair mutations on aminoacylation. Analysis of the loss of $k_{\text {chem }} / K_{\mathrm{d}}$ in logarithmic scale by substitutions of 1-72 in EctRNA ${ }^{\mathrm{fMet}}, E c \mathrm{RNA}{ }^{\mathrm{Met}}$, and EctRNA ${ }^{\mathrm{Cys}}$.
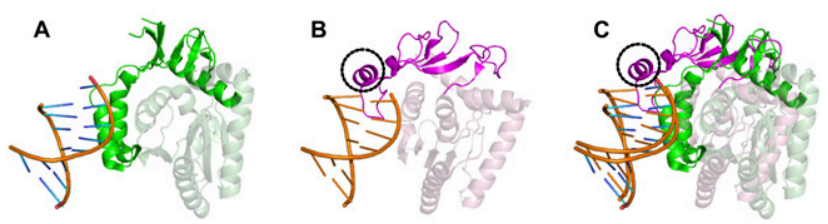

D
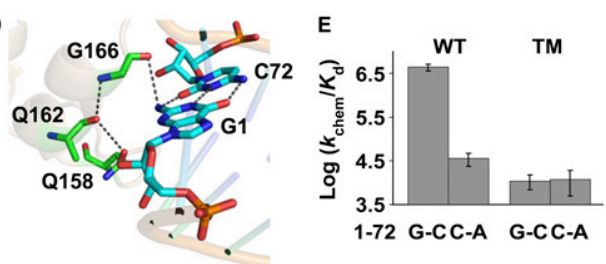

FIGURE 4. Acceptor stem recognition by EcMetRS and EcCysRS. (A) The tRNA-bound structure of A. aeolicus MetRS, showing the CP1 domain of MetRS in green, the Rossmann fold in light green, and tRNA in orange (Protein Data Bank [PDB] identification 2CT8). (B) The tRNA-bound structure of EcCysRS, showing the CP1 domain in magenta, the Rossmann fold in light pink, and tRNA in orange (PDB identification 1U0B). (C) Superimposition of the tRNA-bound EcCysRS and EcMetRS by aligning the $5^{\prime}$ half of tRNA backbones (nucleotides 1-34), revealing the presence of the inserted $\alpha$-helical motif in EcCysRS (circled in black). (D) Interaction between the $\alpha$-helical motif in EcCysRS and the G1-C72 nucleotides in EctRNA ${ }^{\text {Cys }}$. The helical motif is shown in light green, while the tRNA acceptor end is shown in light orange in the background. The residues Q158, Q162, and G166 are highlighted in sticks with carbons in green, while $\mathrm{G} 1$ and $\mathrm{C} 72$ are shown in sticks with carbons in cyan. (E) Effect of the triple mutations (Q158A, Q162A, and G166A) in EcCysRS on aminoacylation of tRNA. The activity is shown in logarithmic scale, while the 1-72 base pairs of the tRNA are indicated at the bottom of the bar graph. (WT) Wild-type EcCysRS; (TM) triple mutant harboring the Q158A, Q162A, and G166A mutations. Graphs showing the structures are generated by PyMol (Delano Scientific).

was unoccupied in AaMetRS (Fig. 4C), suggesting that the motif might be involved in the stringent monitoring of the 1-72 base pair by EcCysRS.

We tested the significance of the $\alpha$-helical motif in $E c$ CysRS by evaluating a triple mutant (TM) enzyme for its ability to stringently discriminate against unpaired 1-72 in the acceptor stem. The mutant enzyme contained alanine substitutions for G166, Q162, and Q158, which are conserved among the CP1 sequences of CysRSs from bacteria to humans. Of the three, G166 in EcCysRS is proximal to the G1-C72 base pair (only $3.5 \AA$ away from the 2-carbonyl of $\mathrm{C} 72$ and $3.7 \AA$ away from $2^{\prime}$-OH of $\mathrm{C} 72$ ), and it stabilizes both the main-chain of Q162 and the 2- $\mathrm{NH}_{2}$ of G1. In turn, Q162 stabilizes the main-chain of Q158, which is proximal to the backbone of G1 (Fig. 4D). These three residues form a $\mathrm{H}$-bonding network that constrains the spatial position of G1, providing an explanation for the importance of G1 and the deleterious effect of the G1C substitution in the mutational analysis (Table 1; Fig. 3). Indeed, single turnover assays showed that in contrast to the WT EcCysRS, the TM had lost the ability to discriminate between G-C and C-A at the 1-72 position. Whereas the WT enzyme discriminated G-C from C-A by 110-fold, 
the mutant exhibited virtually identical values of $k_{\text {chem }} / K_{\mathrm{d}}$ (tRNA) for the two tRNA substrates (Fig. 4E). Notably, while the $k_{\text {chem }} / K_{\mathrm{d}}$ (tRNA) values of the mutant enzyme for the two tRNA substrates were reduced by more than 400 -fold relative to the WT enzyme (Table 2), they were well above the detection limit (as low as $10^{5}$ - to $10^{6}$-fold down) due to the use of high specific activity of ${ }^{35} \mathrm{~S}$-Cys for aminoacylation (Zhang et al. 2008). Thus, mutations of conserved residues in the $\alpha$-helical motif in the CP1 of EcCysRS that docked on 1-72 abolished the ability of the enzyme to stringently monitor the 1-72 structure, supporting the importance of the motif in the recognition of tRNA acceptor end.

\section{DISCUSSION}

The Rossmann fold in the class I aaRSs consists of two halves, each of which is made up of a secondary arrangement of $\beta \alpha \beta \alpha \beta$. Although the two halves have highly conserved structures among the different members of the class I enzymes, it was noted previously that the $\mathrm{CP} 1$ segment that connects the two halves is variable in both sequence and length (Hou et al. 1991). The length variations range from short (64 residues in ArgRS, 69 residues in CysRS) to medium (126 residues in MetRS) to long (273 residues in ValRS, 287 residues in LeuRS, and 300 residues in IleRS) among the E. coli enzymes (Hou et al. 1991). For the three enzymes with a long CP1 (ValRS, LeuRS, and IleRS), a substantial portion of the connective polypeptide is dedicated to a quality-control editing function for the removal of misacylated aa-tRNA (Ibba and Soll 2000). For EcCysRS, despite having one of the shortest CP1 (due in part to the lack of an editing domain) (Fersht and Dingwall 1979), it still manages to fold an $\alpha$-helical motif (from residues 158-166) that docks on the 1-72 base pair. This $\alpha$-helical motif forms an extensive H-bonding network through the conserved Q158, Q162, and G166 to stabilize the backbone of 1-72, providing a basis to monitor the structure at the acceptor end. Interestingly, this helical motif was disordered in the tRNA-free EcCysRS structure (Newberry et al. 2002), but it became organized with the bound tRNA, strongly indicating a role in tRNA recognition. Here we show that a TM that disrupts the H-bonding network in the Q158-Q162-G166 motif eliminates the ability of $E c C y s R S$ to discriminate against C-A at 1-72, consistent with the notion that this motif specifically monitors the tRNA structure at 1-72. In contrast, despite having a longer CP1 relative to EcCysRS, EcMetRS lacks this motif, which can provide an explanation for the relaxed recognition at 1-72.

The placement of a specific structural motif, emanating from the CP1 polypeptide, at a spatial position capable of monitoring the 1-72 structure appears common to other class I aaRSs. Analysis of the tRNA-bound structures for six additional class I enzymes was performed (Fig. 5). All of these six structures retain the tRNA acceptor end in the Rossmann fold, whereas the structures of the remaining two enzymes (ValRS and IleRS) place the end in the editing domain (Silvian et al. 1999; Fukai et al. 2000). Structural alignment of the six structures was performed by superposition of tRNA backbones at nucleotides 1-34, which revealed that in each case there exists a specific structural motif near the 1-72 base pair. This structural motif in TyrRS (Yaremchuk et al. 2002) and TrpRS (Yang et al. 2006) is a long $\alpha$-helix, whereas it is a short helix in GluRS (Sekine et al. 2003). In contrast, the structural motif in ArgRS (Konno et al. 2009) and GlnRS (Bullock et al. 2003) exists as a short loop. In all cases, an equivalent structural motif is absent from AaMetRS.

More detailed analysis revealed how the specific structural motif monitors the base pairing at 1-72 to provide a mechanism to reject incorrect structures (Fig. 6). As a reference, analysis of the structure of AaMetRS reveals that the enzyme contacts only the backbone at position 72 (by the side-chains of Q199 and R262) but has no obvious contact with the first nucleotide (Fig. 6A). In contrast, each of the enzymes described above engages the identified specific motif to recognize the structure of 1-72. We have grouped these enzymes into three mechanisms of recognition. First, CysRS, GlnRS, and TyrRS make contact with the 1-72 backbone not only at position 72 but also at position 1, the latter of which can involve a water-mediated interaction. For example, EcCysRS engages the main-chain of G166 in the $\alpha$-helical insertion motif to contact the $2-\mathrm{NH}_{2}$ of $\mathrm{G} 1$ and the side-chain of $\mathrm{L} 163$ in the $\mathrm{CP} 1$ to contact $2^{\prime}-\mathrm{OH}$ of C72, thus stabilizing both G1 and C72, while additionally it uses F232 and D229 in the Rossmann fold to orient the phosphate of C72 through a water-mediated interaction (Fig. 6B). Similarly, EcGlnRS interacts with both nucleotides at 1-72, using the main-chain of P181 in the Rossmann fold and the side-chain of R133 in the insertion motif to contact

TABLE 2. Effect of amino acid substitution in the helical motif of the CP1 domain of CysRS on aminoacylation

\begin{tabular}{|c|c|c|c|c|c|c|}
\hline Enzyme & tRNA & $\begin{array}{l}\text { Nucleotides } \\
\text { at } 1-72\end{array}$ & $k_{\text {chem }}\left(\sec ^{-1}\right)$ & $K_{\mathrm{d}}(\mu \mathrm{M})$ & $k_{\text {chem }} / K_{\mathrm{d}}\left(\mathrm{sec}^{-1} \mathrm{M}^{-1}\right)$ & $\begin{array}{c}\text { Relative activity } \\
\text { to WT tRNA }\end{array}$ \\
\hline \multirow[t]{2}{*}{$\overline{E c C y s R S ~(W T) ~}$} & tRNA ${ }^{\text {Cys }}(W T)$ & $\mathrm{G}-\mathrm{C}$ & $14 \pm 2$ & $3.2 \pm 0.4$ & $(4.4 \pm 0.8) \times 10^{6}$ & 1 \\
\hline & tRNA ${ }^{\text {Cys }}$ (C1-A72 mutant) & C-A & $0.5 \pm 0.1$ & $14 \pm 4$ & $(4 \pm 1) \times 10^{4}$ & $1 / 110$ \\
\hline \multirow[t]{2}{*}{ EcCysRS (TM) } & tRNA ${ }^{\text {Cys }}(\mathrm{WT})$ & $\mathrm{G}-\mathrm{C}$ & $0.34 \pm 0.07$ & $30 \pm 10$ & $(1.1 \pm 0.4) \times 10^{4}$ & 1 \\
\hline & tRNA ${ }^{\text {Cys }}$ (C1-A72 mutant) & C-A & $0.10 \pm 0.04$ & $8 \pm 3$ & $(1.3 \pm 0.7) \times 10^{4}$ & $1 / 1$ \\
\hline
\end{tabular}

EcCysRS (TM) harbors Q158A, Q162A, and G166A substitutions. The error values represent standard error values. 


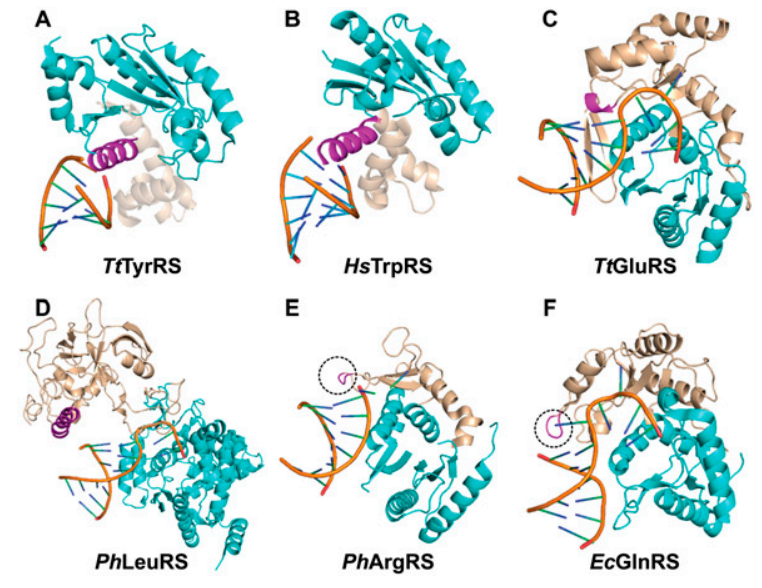

FIGURE 5. Acceptor stem recognition by class I aaRSs. (A) Thermus thermophilus TyrRS (Protein Data Bank [PDB] identification 1H3E); (B) human HsTrpRS (PDB identification 2AZX); (C) T. thermophilus GluRS (PDB identification 1N77); (D) P. horikoshii LeuRS (PDB identification 1WZ2); (E) P. horikoshii ArgRS (PDB identification 2ZUE); and (F) E. coli GlnRS (PDB identification 1O0B). (Cyan) Rossmann fold; (light pink) CP1 domain; (magenta) motif in the CP1 domain that docks on 1-72.

the $2-\mathrm{NH}_{2}$ of $\mathrm{G} 1$ and the $2^{\prime}-\mathrm{OH}$ of $\mathrm{U} 72$, respectively (data not shown). Note that the G1 in the EcGlnRS crystal structure is a replacement of the natural A1 in EctRNA ${ }^{\text {Gln }}$. In the structure of Thermus thermophilus (Tt) TyrRS (Yaremchuk et al. 2002), the side-chains of R205 and R209 of the $\alpha$-helical insertion motif make H-bonds with the phosphates of $\mathrm{G1}$, and the side-chain of E154 from the Rossmann fold contacts N4 of C72 through a H-bond (data not shown). In the second mechanism, we propose that the insertion motif is in the proximity of 1-72 and that it can interact with the backbone of 1-72 during the mutual adaptation of the enzyme and tRNA en route to aminoacylation. For example, while the existing crystal structure reveals that the insertion motif in TtGluRS (Sekine et al. 2003) is placed too far from the H-bonding distance with the 1-72 nucleotides (e.g., E172 of the motif separated from G1 by $\sim 8 \AA$ and $\mathrm{G} 120$ and R116 of the CP1 domain separated from the backbone of C72 by $4-5 \AA$ ) (Fig. 6C), these distances can be shortened as both the enzyme and tRNA move closer to each other for aminoacylation. The existence of the insertion motif provides a realistic landmark for such shortening to occur. A similar case can be made in the structure of Pyrococcus horikoshii ( $P h)$ ArgRS (Konno et al. 2009). In the third mechanism, as exemplified by the crystal structures of PhLeuRS (Fig. 5D; Fukunaga and Yokoyama 2005) and human Homo sapiens (Hs) TrpRS (Yang et al. 2006), enzyme contact is observed only with the first nucleotide. For example, $P h$ LeuRS contacts G1 at the $\gamma$-oxygen of the phosphate by the side-chain of K402 and of H406 in the insertion motif (Fig. 6D), which can be shifted over to contact the nucleotide 72 by minor structural adjustments of the enzyme-tRNA complex. A similar case can be made for HsTrpRS, which contacts G1 at both the base and the phosphate backbone by specific side-chains in the insertion motif. It should be noted that the observed crystal structures of HsTrpRS and PhArgRS lack the CCA sequence in the tRNA, while the observed crystal structure of TtTyrRS lacks the C75 and A76 nucleotides, suggesting that the identified interactions may be modified in a crystal complex with a complete tRNA molecule in each case.

Thus by analysis of available crystal structures of bacterial class I enzymes, we have identified a common mechanism that enables the insertion of a specific motif into the Rossmann fold to dock on 1-72 of the tRNA acceptor end. We suggest that this docking interaction can provide a basis to inspect the structure of the acceptor end for the proper orientation of the $3^{\prime}$ end during aminoacylation. This hypothesis is supported by the work here describing the inability of a docking-defective EcCysRS mutant to discriminate against the incorrect C-A mutation at 1-72. Importantly, the use of a specific insertion motif to monitor the 1-72 base pairing property in the tRNA acceptor end is more widespread than just the class I aaRSs. For example, crystal structures of three class II enzymes (PheRS [Moor et al. 2006], AspRS [Eiler et al. 1999], and ThrRS [Sankaranarayanan et al. 1999]) show enzyme contact with the backbone of both 1-72 nucleotides. Additionally, the bacterial protein synthesis elongation factor EF-Tu, which brings elongator aa-tRNAs to the ribosome, also has an insertion motif that docks on the 1-72 base pair (Nissen et al. 1995, 1999), which can provide a mechanism to discriminate against the $\mathrm{C}-\mathrm{A}$ of the initiator tRNA. The proposed mechanism is also applicable to methionyl-tRNA formylase (MTF), which must select the initiator tRNA ${ }^{\mathrm{fMet}}$ for the formyl transfer to the aminoacyl moiety of MettRNA $^{\text {fMet }}$. The crystal structure of an MTF shows the presence of an enzyme structural loop inserted between the

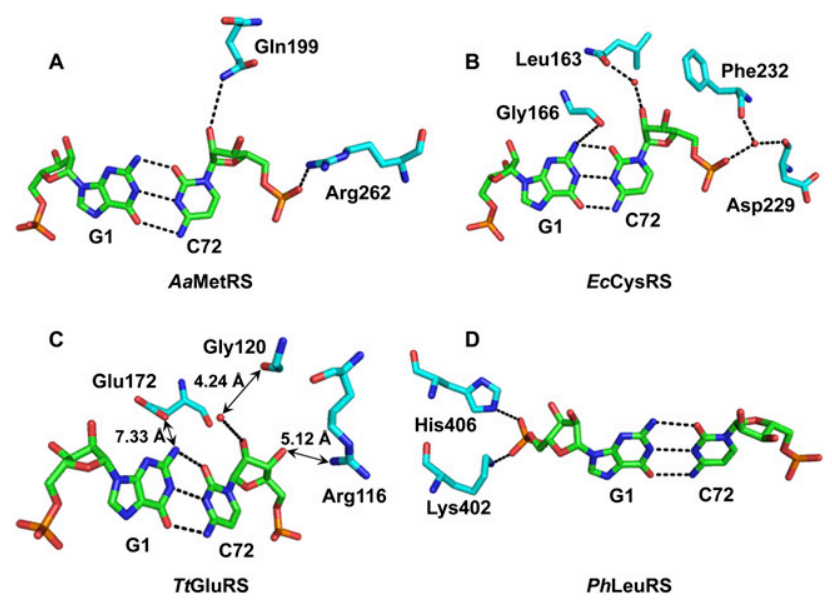

FIGURE 6. Recognition of 1-72 in tRNA by class I aaRSs. (A) Structure of A. aeolicus MetRS (PDB 2CT8); (B) structure of E. coli CysRS (PDB 1U0B); $(C)$ structure of $T$. thermophilus GluRS (PDB 1N77); and (D) structure of P. horikoshii LeuRS (PDB 1WZ2). 
unpaired C-A at 1-72 to exclude recognition of elongator tRNA (Schmitt et al. 1998).

This work demonstrates that the insertion of a specific structural motif in between the two halves of the class I Rossmann fold can provide a mechanism to inspect the 1-72 base pairing interaction of the tRNA acceptor end. While the details of the structural motif can vary (ranging from $\alpha$ helices to loops), depending on the nucleotide sequence near the acceptor end of the cognate tRNA, the motif is invariably placed near the 1-72 position within a distance that can be easily adjusted for direct contact in the enzyme-tRNA complex. The lack of such an insertion motif in bacterial MetRS gives the enzyme the flexibility to recognize both paired and unpaired acceptor end.

\section{MATERIALS AND METHODS}

\section{Plasmids and mutants}

The genes for EcCysRS and EcMetRS were cloned separately into pET22b for expression in E. coli (Zhang et al. 2003). The EcCysRS mutants were constructed using site-directed mutagenesis (Agilent Biotechnologies), and the mutations were confirmed by DNA sequencing. The genes for tRNAs and their mutants were constructed by overlapping oligonucleotides synthesized by IDT and were further extended by sequenase. The forward oligonucleotides for tRNAs that begin with a nonguanosine base contain the gene for a self-cleavable hammerhead ribozyme upstream of the tRNA gene.

\section{Protein expression and purification}

Recombinant enzymes were expressed from the pET22b vector in BL21(DE3) cells at $37^{\circ} \mathrm{C}$ upon induction with $0.4 \mathrm{mM}$ IPTG. The enzymes were purified using Ni-NTA resin following the instructions from the manufacturer and were estimated by SDSPAGE to be $>95 \%$ pure. The enzyme concentrations were measured by Bradford assay with BSA as the standard and were corrected by active site burst assay.

\section{tRNA preparation}

tRNA transcripts were prepared by in vitro transcription with T7 RNA polymerase from double-stranded DNA templates. Transcripts of tRNAs were separated from DNA templates and NTPs by a $12 \%$ PAGE/urea gel, excised, crushed, and soaked in TE (10 mM Tris$\mathrm{HCl}$ at $\mathrm{pH}$ 8.0, $1 \mathrm{mM}$ EDTA). After ethanol precipitation, tRNAs were stored in TE. The concentrations of tRNAs were determined by absorption at $260 \mathrm{~nm}\left(1 \mathrm{OD}_{260}\right.$ unit equivalent to $\left.40 \mu \mathrm{g} / \mathrm{mL}\right)$ and were further corrected by plateau charging.

\section{Pre-steady-state assay for aminoacylation}

The pre-steady-state assays for aminoacylation by EcMetRS was performed on the quench-flow RQF-3 KinTek instrument, using the constant quench option. The reaction buffer contains $20 \mathrm{mM}$ $\mathrm{KCl}, 20 \mathrm{mM}$ Tris- $\mathrm{HCl}$ ( $\mathrm{pH}$ 7.5), $10 \mathrm{mM} \mathrm{MgCl} 2,8 \mathrm{mM}$ ATP, and $500 \mu \mathrm{M}{ }^{3} \mathrm{H}$-methionine (specific activity $315 \mathrm{dpm} / \mathrm{pmol}$ ). MetRS $(1 \mu \mathrm{M})$ was incubated in one syringe and was rapidly mixed with
$10 \mu \mathrm{M}$ tRNA, ATP, and methionine from the other syringe. Reactions were quenched by 5\% TCA, followed by $0.5 \mathrm{mM}$ $\beta$-mercaptoethanol, and product formation of ${ }^{3} \mathrm{H}$-Met-tRNA ${ }^{\text {Met }}$ or ${ }^{3} \mathrm{H}$-Met-tRNA ${ }^{\mathrm{fMet}}$ was monitored by acid precipitation over time. Data were fit to the following burst equation: $y=$ $y_{o}+A \times\left(1-e^{-k_{1} \times t}\right)+k_{2} \times E_{o} \times t$, where $y_{\mathrm{o}}$ is the $y$ intercept, $A$ is the amplitude of the initial exponential phase, $k_{1}$ is the apparent rate constant of the initial exponential regression, $k_{2}$ is the apparent rate constant of the steady-state phase, and $t$ is the time in seconds.

\section{Single-turnover assay for aminoacylation}

Single turnover assay for aminoacylation was performed using the same buffer condition as for the burst assay. The enzyme (1-50 $\mu \mathrm{M}$ ) incubated in one syringe was rapidly mixed with tRNA (1 $\mu \mathrm{M})$, ATP $(8 \mathrm{mM})$, and ${ }^{3} \mathrm{H}$-methionine $(500 \mu \mathrm{M}$, specific activity $315 \mathrm{dpm} / \mathrm{pmole})$ or ${ }^{35} \mathrm{~S}$-cysteine $(500 \mu \mathrm{M}$, specific activity 860 $\mathrm{dpm} / \mathrm{pmol}$ ) from the other syringe. The time courses for product formation of ${ }^{3} \mathrm{H}$-Met-tRNA ${ }^{\text {Met }}$ or ${ }^{3} \mathrm{H}$-Met-tRNA ${ }^{\mathrm{fMet}}$ or ${ }^{35} \mathrm{~S}$-Cys$\mathrm{tRNA}^{\mathrm{Cys}}$ were fit to the single exponential equation: $y=$ $y_{o}+A \times\left(1-e^{-k a p p \times t}\right)$, where $y_{\mathrm{o}}$ is the $y$ intercept, $A$ is the scaling constant, $k_{\text {app }}$ is the apparent rate constant, and $t$ is the time in seconds. The data $k_{\text {app }}$ versus enzyme concentration were fit to the Michaelis-Menton equation: $y=k_{\text {chem }} \times E_{o} /\left(E_{o}+K_{d}\right)$, where $k_{\text {chem }}$ is rate constant for chemistry step and $E_{\mathrm{o}}$ is the enzyme concentration.

\section{Structural analysis of class I aaRSs}

The crystal structures of class I aaRS-tRNA complexes were analyzed by using PyMOL (Delano Scientific). Each available structure was aligned with AaMetRS-tRNA ${ }^{\text {Met }}$ complex (2CT8) by superimposing the $5^{\prime}$ half (1-34) of the tRNA backbones. This was used to identify specific structural motifs that can monitor the 1-72 base pair while missing from the structure of MetRS. Further detailed analysis focused on the residues in each aaRSs that contact the 1-72 nucleotides in the tRNA substrate.

\section{SUPPLEMENTAL MATERIAL}

Supplemental material is available for this article.

\section{ACKNOWLEDGMENTS}

This work is supported by NIH GM081601 to Y.M.H. We thank Eric First, John Perona, and Susan Martinis for discussion.

Received August 18, 2011; accepted November 9, 2011.

\section{REFERENCES}

Bullock TL, Uter N, Nissan TA, Perona JJ. 2003. Amino acid discrimination by a class I aminoacyl-tRNA synthetase specified by negative determinants. J Mol Biol 328: 395-408.

Eiler S, Dock-Bregeon A, Moulinier L, Thierry JC, Moras D. 1999. Synthesis of aspartyl-tRNA(Asp) in Escherichia coli: a snapshot of the second step. EMBO J 18: 6532-6541.

Eldred EW, Schimmel PR. 1972. Investigation of the transfer of amino acid from a transfer ribonucleic acid synthetase-aminoacyl adenylate complex to transfer ribonucleic acid. Biochemistry 11: 17-23. 
Fechter P, Rudinger J, Giege R, Theobald-Dietrich A. 1998. Ribozyme processed tRNA transcripts with unfriendly internal promoter for T7 RNA polymerase: production and activity. FEBS Lett 436: 99-103.

Fersht AR, Dingwall C. 1979. Cysteinyl-tRNA synthetase from Escherichia coli does not need an editing mechanism to reject serine and alanine. High binding energy of small groups in specific molecular interactions. Biochemistry 18: 1245-1249.

Fukai S, Nureki O, Sekine S, Shimada A, Tao J, Vassylyev DG, Yokoyama S. 2000. Structural basis for double-sieve discrimination of L-valine from L-isoleucine and L-threonine by the complex of tRNA(Val) and valyl-tRNA synthetase. Cell 103: 793-803.

Fukunaga R, Yokoyama S. 2005. Aminoacylation complex structures of leucyl-tRNA synthetase and tRNA(Leu) reveal two modes of discriminator-base recognition. Nat Struct Mol Biol 12: 915-922.

Gillet S, Hountondji C, Schmitter JM, Blanquet S. 1997. Covalent methionylation of Escherichia coli methionyl-tRNA synthethase: identification of the labeled amino acid residues by matrix-assisted laser desorption-ionization mass spectrometry. Protein Sci 6: 2426-2435.

Guth E, Connolly SH, Bovee M, Francklyn CS. 2005. A substrateassisted concerted mechanism for aminoacylation by a class II aminoacyl-tRNA synthetase. Biochemistry 44: 3785-3794.

Hauenstein S, Zhang CM, Hou YM, Perona JJ. 2004. Shape-selective RNA recognition by cysteinyl-tRNA synthetase. Nat Struct Mol Biol 11: 1134-1141.

Hou YM, Shiba K, Mottes C, Schimmel P. 1991. Sequence determination and modeling of structural motifs for the smallest monomeric aminoacyl-tRNA synthetase. Proc Natl Acad Sci 88: 976-980.

Hou YM, Lipman RS, Zarutskie JA. 1998. A tRNA circularization assay: evidence for the variation of the conformation of the CCA end. RNA 4: 733-738.

Hou YM, Zhang X, Holland JA, Davis DR. 2001. An important 2'-OH group for an RNA-protein interaction. Nucleic Acids Res 29: 976-985.

Ibba M, Soll D. 2000. Aminoacyl-Trna synthesis. Annu Rev Biochem 69: 617-650.

Ibba M, Morgan S, Curnow AW, Pridmore DR, Vothknecht UC, Gardner W, Lin W, Woese CR, Soll D. 1997. A euryarchaeal lysyl-tRNA synthetase: resemblance to class I synthetases. Science 278: 1119-1122.

Konno M, Sumida T, Uchikawa E, Mori Y, Yanagisawa T, Sekine S, Yokoyama S. 2009. Modeling of tRNA-assisted mechanism of Arg activation based on a structure of Arg-tRNA synthetase, tRNA, and an ATP analog (ANP). FEBS J 276: 4763-4779.

Lee CP, Mandal N, Dyson MR, RajBhandary UL. 1993. The discriminator base influences tRNA structure at the end of the acceptor stem and possibly its interaction with proteins. Proc Natl Acad Sci 90: 7149-7152.

Liu C, Gamper H, Shtivelband S, Hauenstein S, Perona JJ, Hou YM. 2007. Kinetic quality control of anticodon recognition by a eukaryotic aminoacyl-tRNA synthetase. J Mol Biol 367: 1063-1078.

Liu C, Gamper H, Liu H, Cooperman BS, Hou YM. 2011. Potential for interdependent development of tRNA determinants for aminoacylation and ribosome decoding. Nat Commun 2: 329. doi: 10.1038/ncomms1331.

Martinis SA, Schimmel P. 1992. Enzymatic aminoacylation of sequence-specific RNA minihelices and hybrid duplexes with methionine. Proc Natl Acad Sci 89: 65-69.

Moor N, Kotik-Kogan O, Tworowski D, Sukhanova M, Safro M. 2006. The crystal structure of the ternary complex of phenylalanyltRNA synthetase with tRNAPhe and a phenylalanyl-adenylate analogue reveals a conformational switch of the CCA end. Biochemistry 45: 10572-10583.
Mulvey RS, Fersht AR. 1978. Mechanism of aminoacylation of transfer RNA. A pre-steady-state analysis of the reaction pathway catalyzed by the methionyl-tRNA synthetase of Bacillus stearothermophilus. Biochemistry 17: 5591-5597.

Nakanishi K, Ogiso Y, Nakama T, Fukai S, Nureki O. 2005. Structural basis for anticodon recognition by methionyl-tRNA synthetase. Nat Struct Mol Biol 12: 931-932.

Newberry KJ, Hou YM, Perona JJ. 2002. Structural origins of amino acid selection without editing by cysteinyl-tRNA synthetase. EMBO J 21: 2778-2787.

Nissen P, Kjeldgaard M, Thirup S, Polekhina G, Reshetnikova L, Clark BF, Nyborg J. 1995. Crystal structure of the ternary complex of Phe-tRNAPhe, EF-Tu, and a GTP analog. Science 270: 14641472.

Nissen P, Thirup S, Kjeldgaard M, Nyborg J. 1999. The crystal structure of Cys-tRNACys-EF-Tu-GDPNP reveals general and specific features in the ternary complex and in tRNA. Structure 7: 143-156.

Puglisi EV, Puglisi JD, Williamson JR, RajBhandary UL. 1994. NMR analysis of tRNA acceptor stem microhelices: discriminator base change affects tRNA conformation at the $3^{\prime}$ end. Proc Natl Acad Sci 91: 11467-11471.

Sankaranarayanan R, Dock-Bregeon AC, Romby P, Caillet J, Springer M, Rees B, Ehresmann C, Ehresmann B, Moras D. 1999. The structure of threonyl-tRNA synthetase-tRNA(Thr) complex enlightens its repressor activity and reveals an essential zinc ion in the active site. Cell 97: 371-381.

Schmitt E, Moulinier L, Fujiwara S, Imanaka T, Thierry JC, Moras D. 1998. Crystal structure of aspartyl-tRNA synthetase from Pyrococcus kodakaraensis KOD: archaeon specificity and catalytic mechanism of adenylate formation. EMBO J 17: 5227-5237.

Sekine S, Nureki O, Dubois DY, Bernier S, Chenevert R, Lapointe J, Vassylyev DG, Yokoyama S. 2003. ATP binding by glutamyl-tRNA synthetase is switched to the productive mode by tRNA binding. EMBO J 22: 676-688.

Silvian LF, Wang J, Steitz TA. 1999. Insights into editing from an iletRNA synthetase structure with tRNAile and mupirocin. Science 285: 1074-1077.

Uter NT, Perona JJ. 2004. Long-range intramolecular signaling in a tRNA synthetase complex revealed by pre-steady-state kinetics. Proc Natl Acad Sci 101: 14396-14401.

Wolfson AD, Pleiss JA, Uhlenbeck OC. 1998. A new assay for tRNA aminoacylation kinetics. RNA 4: 1019-1023.

Yang XL, Otero FJ, Ewalt KL, Liu J, Swairjo MA, Kohrer C, Rajbhandary UL, Skene RJ, McRee DE, Schimmel P. 2006. Two conformations of a crystalline human tRNA synthetase-tRNA complex: implications for protein synthesis. EMBO J 25: 29192929.

Yaremchuk A, Kriklivyi I, Tukalo M, Cusack S. 2002. Class I tyrosyltRNA synthetase has a class II mode of cognate tRNA recognition. EMBO J 21: 3829-3840.

Zhang CM, Christian T, Newberry KJ, Perona JJ, Hou YM. 2003. Zinc-mediated amino acid discrimination in cysteinyl-tRNA synthetase. J Mol Biol 327: 911-917.

Zhang CM, Perona JJ, Ryu K, Francklyn C, Hou YM. 2006. Distinct kinetic mechanisms of the two classes of aminoacyl-tRNA synthetases. J Mol Biol 361: 300-311.

Zhang CM, Liu C, Slater S, Hou YM. 2008. Aminoacylation of tRNA with phosphoserine for synthesis of cysteinyl-tRNA(Cys). Nat Struct Mol Biol 15: 507-514. 

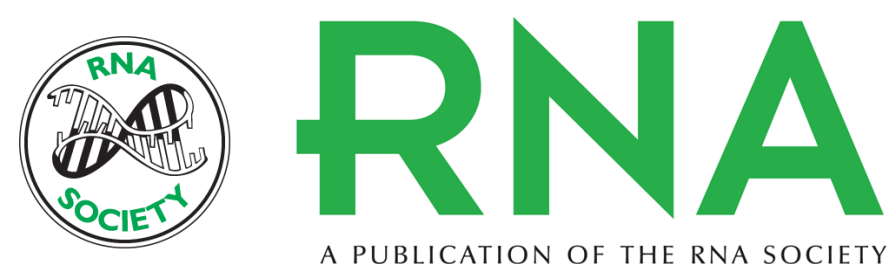

A PUBLICATION OF THE RNA SOCIETY

\section{Adaptation to tRNA acceptor stem structure by flexible adjustment in the catalytic domain of class I tRNA synthetases}

Cuiping Liu, Jeffrey M. Sanders, John M. Pascal, et al.

RNA 2012 18: 213-221 originally published online December 19, 2011

Access the most recent version at doi:10.1261/rna.029983.111

Supplemental
Material http://rnajournal.cshlp.org/content/suppl/2011/12/02/rna.029983.111.DC1

References This article cites 38 articles, 14 of which can be accessed free at:

http://rnajournal.cshlp.org/content/18/2/213.full.html\#ref-list-1

License

Email Alerting Receive free email alerts when new articles cite this article - sign up in the box at the Service top right corner of the article or click here.

To subscribe to $R N A$ go to:

http://rnajournal.cshlp.org/subscriptions 